\title{
Variação temporal da macrofauna bentônica sublitoral da praia da Urca (RJ) após a ocorrência de ressacas
}

\author{
(Temporal variation of the sublittoral benthic macrofauna in Urca Beach (RJ) after storms)
}

\author{
Maria Claudia Rayol Sola \& Paulo Cesar Paiva \\ Instituto de Biologia da Universidade Federal do Rio de Janeiro \\ Departamento de Zoologia \\ (CCS - Bloco A, Ilha do Fundão 21940-590, Rio de Janeiro, RJ, Brasil)
}

A macrofauna bentônica caracteriza-se por ter uma mobilidade muito restrita, fazendo com que seja mantida pelas condições ambientais vigentes $\mathrm{e}$ portanto funcionando, na maioria dos casos, como um excelente indicador biológico em estudos de monitoramento ambiental costeiro. Esta característica se deve ainda ao fato de que os impactos ambientais mais comuns no ambiente costeiro, como a poluição orgânica e inorgânica e a construção de marinas e emissários submarinos, tem como depositório final ou alteração principal as comunidades de sedimentos (Keough \& Quinn, 1991). Não obstante, em estudos ecológicos e oceanográficos de cunho temporal, grandes perturbações hidrodinâmicas tem sido relevadas a um segundo plano. Os padrões de densidade, diversidade, riqueza e de variação temporal das principais espécies têm sido avaliados em função de parâmetros ambientais obtidos concomitantemente.

Correlações observadas entre tais padrões são então interpretadas a luz de parâmetros abióticos e bióticos, como temperatura, salinidade e produção primária, entre outros, enquanto o hidrodinamismo é desconsiderado ou indiretamente estimado a partir da profundidade. Tais análises não consideram portanto a ação de ondas de superfície, principalmente quando se considera aquelas geradas por frentes frias de grande intensidade, não previsíveis e com padrão sazonal pouco definido (Paiva, 1996).

$\mathrm{O}$ efeito das perturbações hidrodinâmicas na macrofauna bentônica tem sido objeto de algumas revisões, destacando-se as de Probert (1984), Butman (1987) e de Hall (1994). Diferentes espécies e principalmente diferentes grupos taxonômicos apresentam respostas distintas em função do seu hábito de vida, mobilidade e capacidade de suportar o impacto físico, com alguns grupos ou espécies podendo inclusive se manter em suspensão durante o período de maior influência das ondas (Grant, 1980; Tamaki, 1987). Não obstante, ainda são escassas informações quanto às alterações na estabilidade estrutural da comunidade refletidas a longo prazo e quais as conseqüências de ressacas extremamente intensas de longa periodicidade.
Com o objetivo de avaliar o papel destas ondas excepcionais, usualmente denominadas como ressacas, foi iniciado um monitoramento da abundância e da dominância da macrofauna bentônica dois dias após uma ressaca de grande intensidade ocorrida em 2 de junho de 1997 e considerada como uma das mais intensas dos últimos 30 anos na Baía da Guanabara. Durante o período foram registradas mais duas ressacas (9 de agosto e 4 de outubro de 1997), porém de intensidade menor que a de junho. Desta forma, o acompanhamento da fauna após o revolvimento do fundo permite uma análise das respostas dos diferentes grupos taxonômicos à este fator. Este revolvimento se daria devido a ação de ondas de grande tamanho causadas por tempestades associadas a sistemas de frentes frias.

O trabalho foi realizado na Praia da Urca $\left(22^{\circ} 57,1^{\prime} \mathrm{S}-43^{\circ} 09,9^{\prime} \mathrm{W}\right)$ no setor oeste da Baía da Guanabara em uma profundidade de cerca de 5 metros. Foram 12 coletas quinzenais (excetuando-se as três primeiras que foram semanais) a partir de 04 de junho de 1997 até 21 de outubro 1997. As datas de todas as coletas são apresentadas na Tabela 1 . Em uma área aproximada de $200 \mathrm{~m}^{2}$ foram distribuídas 10 réplicas tomadas com um amostrador cilíndrico de $100 \mathrm{~mm}$ de diâmetro e $150 \mathrm{~mm}$ de altura (perfazendo um volume de 1,2 litros) através de mergulho autônomo. Após a lavagem das amostras estas foram peneiradas em malha de $0,5 \mathrm{~mm}$ e fixadas em formalina a $10 \%$. Além das amostras biológicas foram tomadas amostras de sedimento para análise granulométrica seguindo os procedimentos sugeridos em Suguio (1973). A macrofauna foi posteriormente triada à nível de grandes grupos taxonômicos em laboratório sob microscópio estereoscópico e quantificada. Os resultados foram analisados através de análise de variância unifatorial (Sokal \& Rohlf, 1995) com a hipótese nula de homogeneidade temporal da densidade dos grupos taxonômicos. As densidades foram logaritimizadas $(\log x+1)$ para normalização. Após feita a análise foram efetuadas comparações entre as diferentes coletas através do teste de Tukey (Sokal \& Rohlf, op. cit.). 
Tabela 1. Datas das amostragens. As datas em negrito foram as primeiras após as três ressacas avaliadas.

\begin{tabular}{cc}
\hline COLETA & DATA \\
\hline $\mathbf{1}$ & $\mathbf{0 4 / 0 6 / 9 7}$ \\
2 & $11 / 06 / 97$ \\
3 & $\mathbf{1 8 / 0 6 / 9 7}$ \\
4 & $03 / 07 / 97$ \\
5 & $16 / 07 / 97$ \\
6 & $30 / 07 / 97$ \\
$\mathbf{7}$ & $\mathbf{1 3 / 0 8 / 9 7}$ \\
8 & $27 / 08 / 97$ \\
9 & $10 / 09 / 97$ \\
10 & $26 / 09 / 97$ \\
$\mathbf{1 1}$ & $\mathbf{0 8 / 1 0 / 9 7}$ \\
12 & $21 / 10 / 97$ \\
\hline
\end{tabular}

\section{Resultados}

Foram coletadas 120 réplicas no total. Nestas foram computados 17 grupos taxonômicos, sendo mais abundantes os seguintes: poliquetas, gamarídeos, ostrácodes, tanaidáceos, cnidários, caprelídeos e cumáceos.

Os poliquetas foram o grupo mais representativo com um crescimento constante após a grande ressaca do dia 02/06/97 (Fig. 1) apresentando um padrão significativo de aumento entre a primeira e as demais coletas e uma queda da nona para a décima coleta, voltando a estabilizar nas coletas seguintes $\left(11^{\mathrm{a}}\right.$ e $\left.12^{\mathrm{a}}\right)$. Os gamarídeos apresentaram um decréscimo significativo a partir da primeira coleta (após a ressaca) se mantendo estáveis depois disto, voltando a decrescer na coleta de número $10 \mathrm{e}$ tornando a estabilizar após esta. A coleta de número 11 ocorreu quatro dias após a terceira ressaca, influenciando o padrão destes animais de maneira semelhante a primeira ressaca de grande intensidade. Tanaidáceos apresentaram valores significativos com decréscimo na $3^{\mathrm{a}}, 8^{\mathrm{a}}$ e $10^{\mathrm{a}}$ coleta, apresentando uma recuperação a partir da $11^{\mathrm{a}}$, demonstrando um comportamento semelhante aos gamarídeos em relação à ressaca. Cumáceos e caprelídeos apresentaram decréscimo significativo após a primeira coleta com um pico na $9^{\text {a }}$ coleta e um decréscimo a partir da $10^{\mathrm{a}}$, voltando a subir na $12^{\mathrm{a}}$. Os cnidários, representados por duas espécies do gênero Renilla ( $R$. reniformes e $R$. muelleri), foram o grupo que apresentou padrão mais diverso, com picos de abundância na $4^{\mathrm{a}}, 5^{\mathrm{a}}, 9^{\mathrm{a}}$ e $12^{\mathrm{a}}$ coletas. Ostrácodes obtiveram padrões temporais não significativos, devido a terem apresentado uma estabilidade ao longo do período de estudo.

Os padrões observados em gamarídeos e caprelídeos, com um pico após a ressaca e um decréscimo ao longo do tempo, podem ser devidos a estes animais serem provenientes dos costões rochosos adjacentes, também afetados pela ressaca. Estes organismos, que vivem associados à fauna incrustante, seriam colocados em suspensão na coluna d'água durante a ressaca depositando-se posteriormente no sedimento, embora sabe-se que existam espécies de gamarídeos que são típicas de sedimento. Este tipo de transporte passivo foi observado por Grant (1980) em gamarídeos devido à perturbação por ondas ou marés excepcionais. Além disso, embora também existam espécies de caprelídeos de sedimento (Ruffo, 1998), estes animais são geralmente adaptados à vida fital, devido a ausência de pleópodes e à adaptação dos três últimos pereiópodes para a fixação principalmente em algas (McLaughlin, 1980). Corroborando esta hipótese de transporte passivo, Colman \& Segrove (1955) e Jansson \& Kallander (1968), encontraram uma abundância de formas bentônicas de peracáridos em amostras tomadas na coluna d'água, embora não tenham sugerido algum fator responsável por tal migração. Contudo, a identificação específica dos anfípodes coletados elucidará melhor à questão do transporte passivo, uma vez que nos permitirá reconhecer o hábito de vida de cada espécie.

O padrão apresentado pelos poliquetas, de certa forma contrário ao dos peracáridos, com baixos valores após a ressaca e um aumento subseqüente ao longo do tempo parece ser uma resposta comum desse táxon para distúrbios físicos sobre o fundo. Segundo Tamaki (1987) o transporte passivo pela ação das ondas e a influência das correntes foram os fatores físicos determinantes na distribuição de alguns grupos habitantes da fauna de sedimentos, principalmente poliquetas. Oliver et al. (1980 apud Hall, 1994) constataram em um estudo sobre a distribuição da fauna de poliquetas e sua relação com distúrbios físicos causados por ondas e correntes na costa da Califórnia (EUA), que no inverno ocorre um decréscimo no número destes animais, pois é quando $a$ atividade das ondas é mais intensa.

Paiva (1994) levantou a hipótese de que a distribuição diferenciada da fauna de poliquetas da plataforma continental do litoral norte paulista seria condicionada pela maior ou menor exposição aos sistemas de frentes frias e as ondas a estes associados. Segundo o mesmo autor, em áreas rasas, o freqüente revolvimento do fundo faz com que haja uma dominância de espécies pequenas de superfície, as quais colonizariam estas áreas no intervalo entre tempestades. As espécies de subsuperfície, não seriam afetadas, já que o revolvimento de ondas atingiria principalmente a superfície do sedimento.

Como as larvas de muitos poliquetas e bivalves tem um comportamento hidrodinâmico semelhante à partículas finas (Butman, 1987), a variação temporal da granulometria poderia refletir os padrões observados para estes táxons. Para tanto, 
foram feitas análises granulométricas referentes aos dias 04/06/97 (coleta 1- após a ressaca), 16/07/97 (coleta 5) e 03/12/97 (após a última coleta). O padrão de granulometria encontrado na praia da Urca (Fig. 2) foi basicamente areia média com um percentual superior a $50 \%$ em todas as amostras, independente do efeito das ressacas. As frações mais finas do sedimento, representadas por areia fina, areia muito fina e lama, sofreram uma maior influência do hidrodinamismo, provavelmente sendo revolvidas pela ação das ondas e voltando a se depositar ao longo do tempo após a passagem da ressaca. Este dado corrobora de certa forma a hipótese de um assentamento comum de sedimentos finos e larvas de poliquetas (Butman, 1987) levando a um incremento da densidade de poliquetas devido ao recrutamento após a ressaca.

\section{POLYCHAETA}

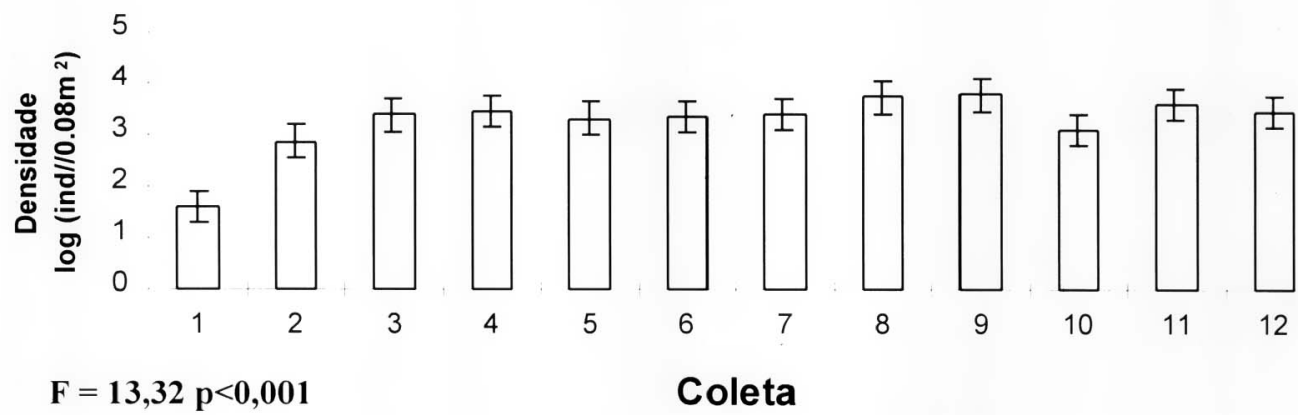

GAMMARIDEA
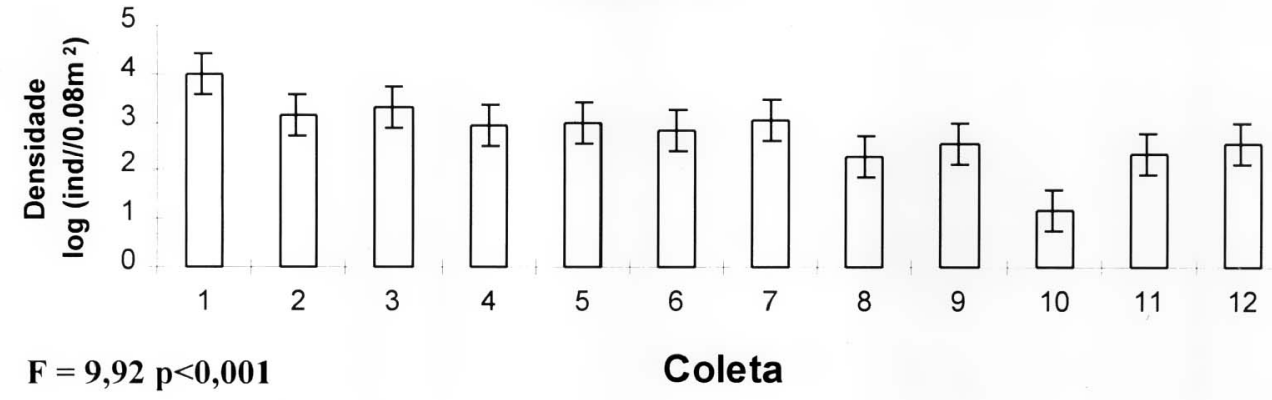

Coleta

TANAIDACEA
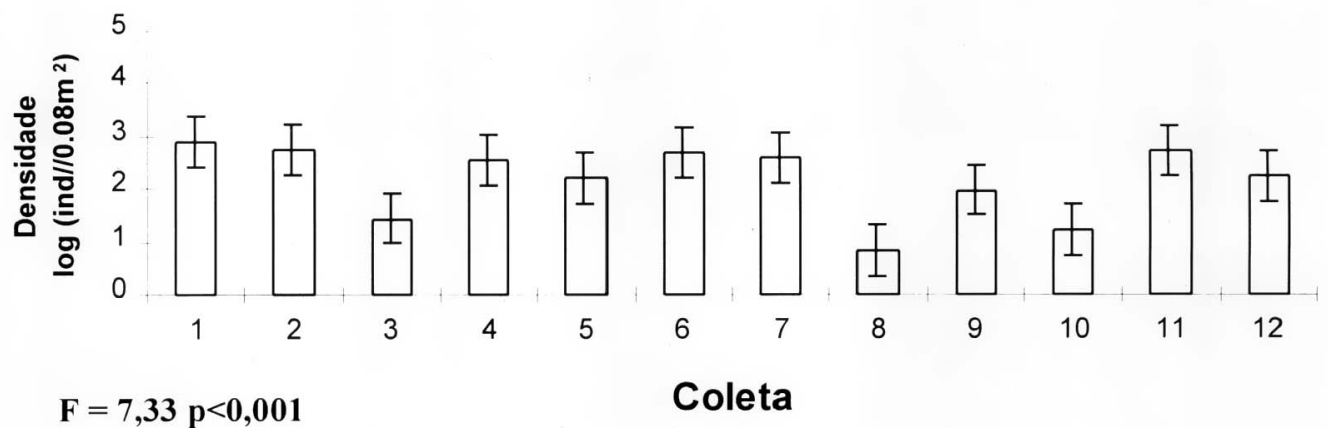

\section{Coleta}

Fig. 1. Variação temporal da densidade dos principais grupos taxonômicos. São apresentados os resultados da ANOVA e do teste à posteriori de Tukey na forma de barras de erro. 
OSTRACODA

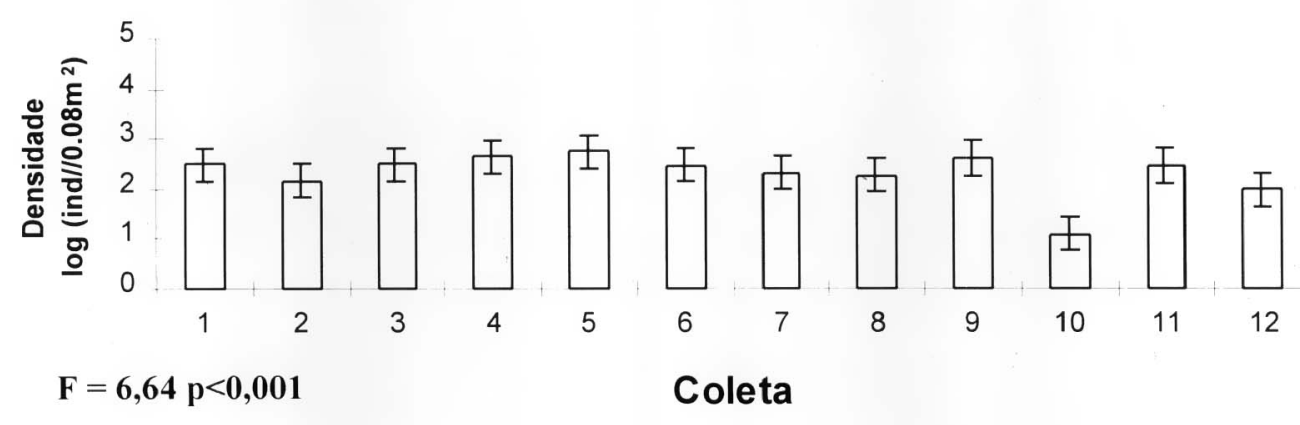

CUMACEA

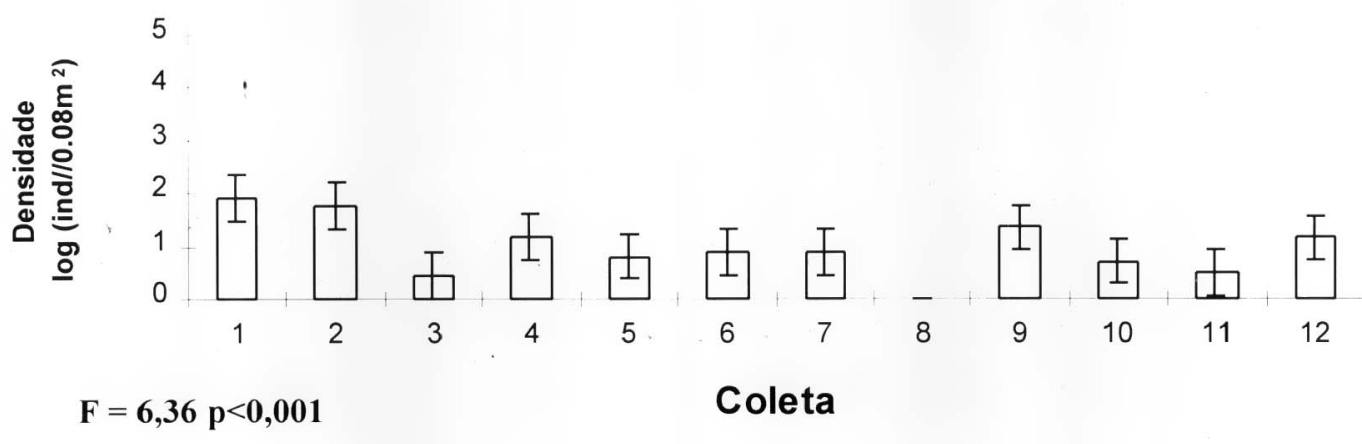

CAPRELLIDAE

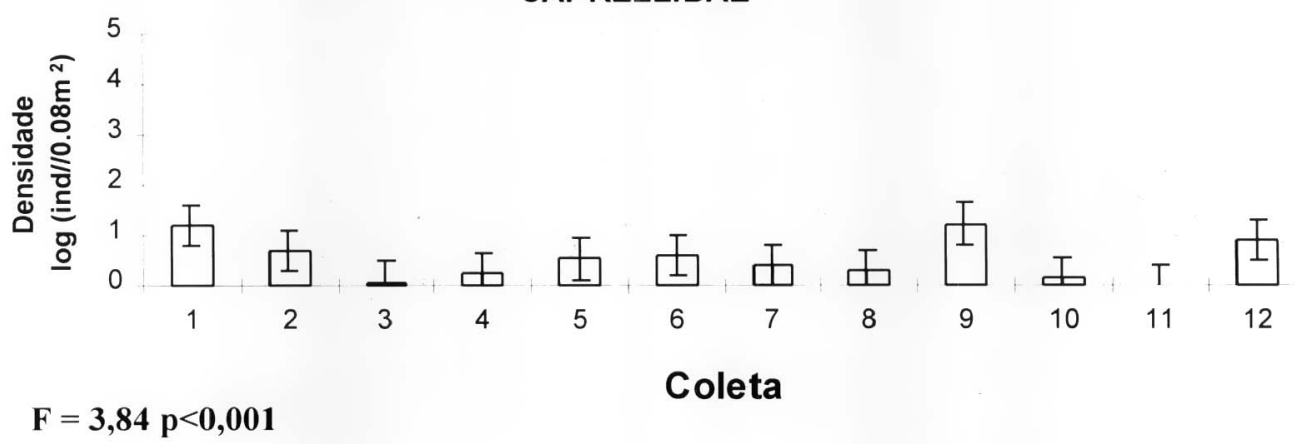

CNIDARIA

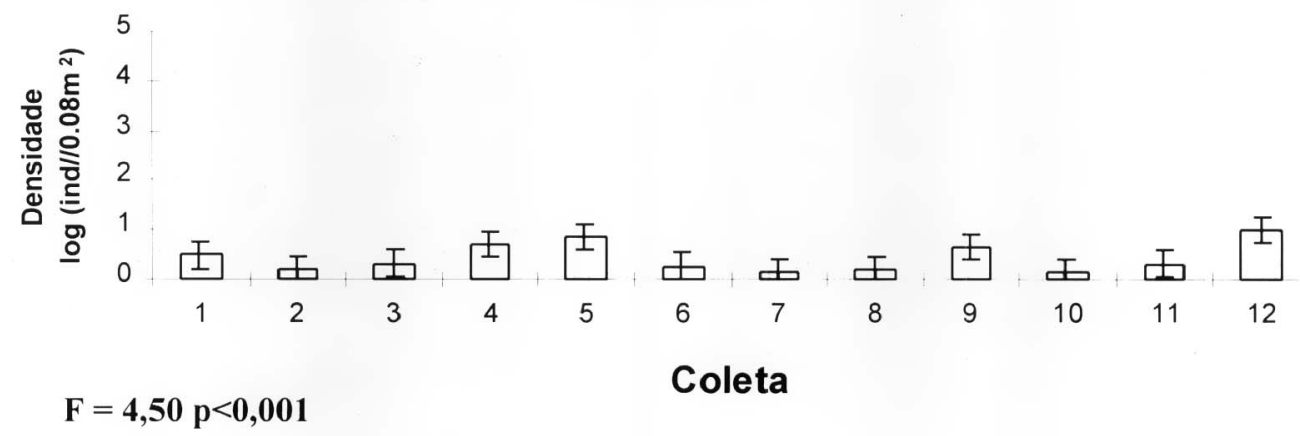

Fig. 1. Cont. 


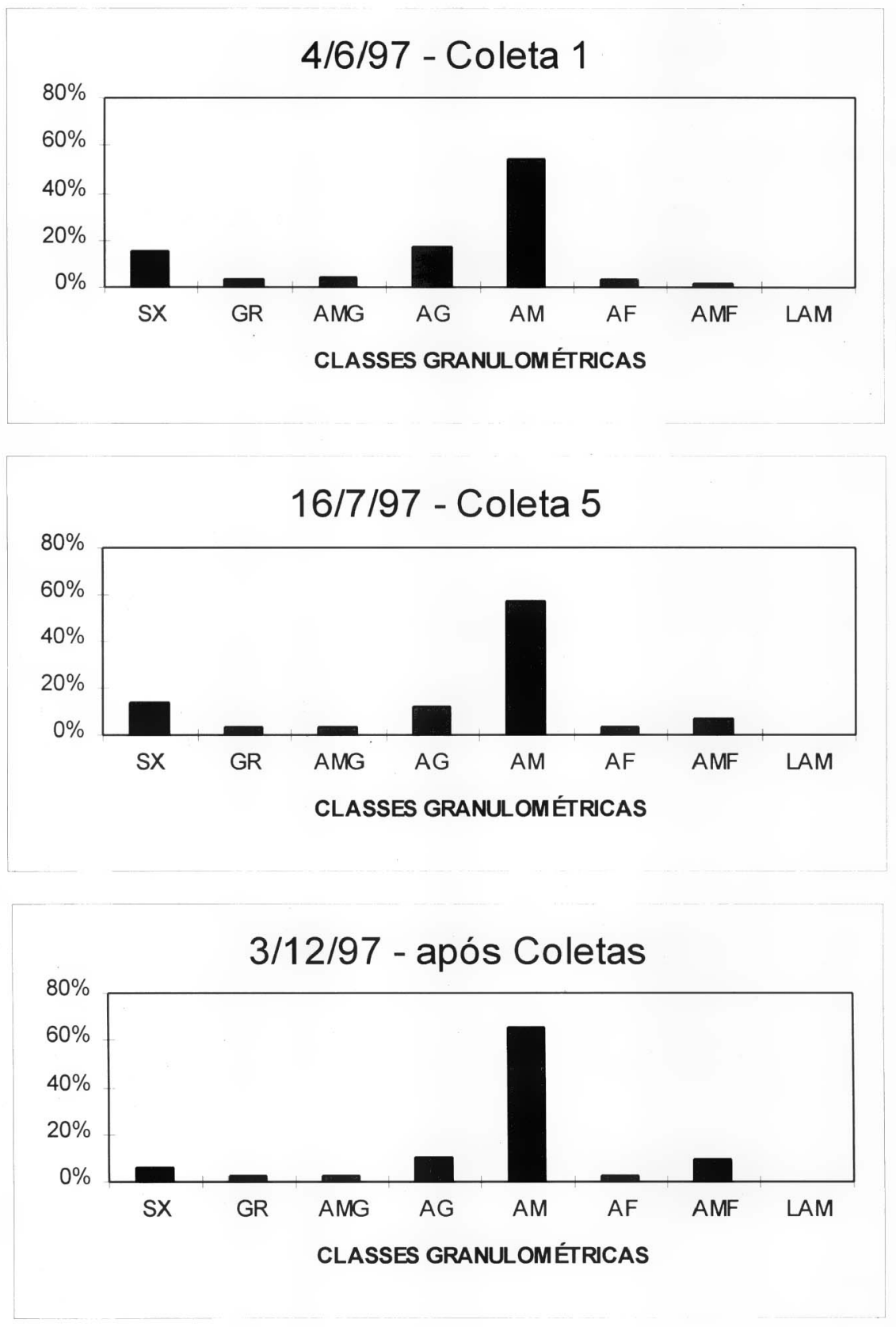

Fig. 2. Composição granulométrica do sedimento ao longo do tempo. SX = seixo; GR = grânulo; $\mathrm{AMG}=$ areia muito grossa; $\mathrm{AG}=$ areia grossa; $\mathrm{AM}=$ areia média; $\mathrm{AF}=$ areia fina; $\mathrm{AMF}=$ areia muito fina; $\mathrm{LAM}=$ lama.

$O$ efeito da ressaca aparentemente não modificou o tipo de sedimento a longo prazo, apesar da metodologia de amostragem não permitir avaliar alterações quanto à estratificação do mesmo. Não obstante o efeito hidrodinâmico da ressaca parece ser direto sobre a fauna e não um efeito indireto sobre o sedimento com conseqüências na estrutura faunística, conforme propunha a hipótese da relação animalsedimento, muito criticada por Butman (1987) ao revisar o papel dos processos hidrodinâmicos no assentamento larval e na estruturação da macrofauna bentônica.

\section{Agradecimentos}

À FAPERJ (Fundação de Amparo a Pesquisa do Estado do Rio de Janeiro) pela concessão da bolsa de apoio técnico a primeira autora (processo: E26/150.182/97), à Dra. Cristiana Serejo (Museu Nacional - UFRJ) pelo auxílio com a bibliografia sobre peracáridos, ao Dr. Paulo Secchin Young (Museu Nacional - UFRJ) pelo auxílio na identificação dos ostrácodes, e à todos aqueles colegas da UFRJ que colaboraram nas laboriosas atividades de campo: Joana Zanol, Christine Ruta, 
Priscilla March, Carlos Alejandro Echeverría, Marcelo Semeraro e Bárbara Segal Ramos.

\section{Referências bibliográficas}

Butman, C. A. 1987. Larval settlement of softsediment invertebrates: the spatial scales of pattern explained by active habitat selection and the emerging role of hydrodynamical processes. Oceanogr. mar. Biol. a. Rev., 25:113-165.

Colman, J. S. \& Segrove, F. 1955. The tidal plankton over stoupe beck sands, Robin Hood's bay (Yorkshire, North Riding). J. Anim. Ecol., 24(2):445-462.

Grant, J. 1980. A flume study of drift in marine infaunal amphipods (Haustoriidae). Mar. Biol., $56(1): 79-84$.

Hall, S. J. 1994. Physical disturbance and marine benthic communities: life in unconsolidated sediments. Oceanogr. mar. Biol. a. Rev., 32:179239.

Jansson, B. O. \& Kallander, C. 1968. On the diurnal activity of some littoral peracarid crustaceans in the Baltic Sea. J. exp. mar. Biol. Ecol., 2:24-36.

Keough, M. J. \& Quinn, G. P. 1991. Causality and the choice of measurements for detecting human impacts in marine enviroments. Aust. J. mar. Freshwat Res., 42(5):539-554.

McLaughlin, P. A. 1980. Comparitive morphology of recent Crustacea. W. H. Freeman, San Francisco, Company. 177p.
Paiva, P. C. 1994. Trophic structure of a shelf polychaete taxocoenosis in southern Brazil. Cah. Biol. mar. 35(1):39-55.

Paiva, P. C. 1996. Variação espacial e temporal da macrofauna bentônica da enseada de Picinguaba, Ubatuba, SP: relevância no planejamento amostral em estudos oceanográficos e de monitoramento ambiental de fundos marinhos inconsolidados. $\mathrm{PhD}$ thesis. Universidade de São Paulo, Instituto Oceanográfico. 140p.

Probert, P. K. 1984. Disturbance, sediment stability and trophic structure of soft-botton communities. J. mar. Res., 42(4):893-921.

Ruffo, S. 1998. The Amphipoda of Mediterranean. Part 4. Mém. Inst. oceanogr. Monaco, 13:1-959.

Sokal, R. R. \& Rohlf, F. J. 1995. Biometry: the principles and practice of statistics in geological research. New York, W. H. Freeman and Company. 887p.

Suguio, K. 1973. Introdução a sedimentologia. São Paulo, Blücher/EDUSP, 317p.

Tamaki, A. 1987. Comparision of resistivity to transport by wave action in several polychaete species on an intertidal sand flat. Mar. Ecol. Prog. Ser., 37(2-3):181-189.

(Manuscript received 17 December 1999; revised 17 December 2001; accepted 07 February 2002) 